\title{
A Comparison of Disability Weights for Alcohol Use Disorders
}

\author{
Jiraluck Nontarak, M.Sc. ${ }^{1,2}$, Sawitri Assanangkornchai, M.D., Ph.D. ${ }^{2}$, Sarah Callinan, Ph.D. ${ }^{3}$ \\ ${ }^{1}$ Health Systems Research Institute, Mueang, Nonthaburi 11000, Thailand. \\ ${ }^{2}$ Department of Epidemiology, Faculty of Medicine, Prince of Songkla University, Hat Yai, Songkhla 90110, Thailand. \\ ${ }^{3}$ Centre for Alcohol Policy Research, Latrobe University, Victoria 3083, Australia. \\ Received 21 February 2020 • Revised 22 May 2020 • Accepted 3 June 2020 • Published online 24 August 2020
}

\section{Abstract:}

Objective: This study aims to determine and compare the disability weights of alcohol use disorders (AUD) based on responses from AUD patients and a non-patient population using three valuation methods.

Material and Methods: Cross-sectional data were collected from three hospitals in southern Thailand. Two groups of participants were recruited: 150 patients diagnosed with AUD and a control group containing 150 persons without AUD. Both groups were asked to rate the AUD health states using a visual analogue scale (VAS), and again using either the European Quality of Life-5 Dimension (EQ-5D) instrument or the time trade-off (TTO) technique. Data were collected via face-to-face interviews.

Results: The mean disability weights, based on the VAS, TTO and EQ-5D valuation methods obtained from AUD patients were: $0.485,0.405$, and 0.311 , respectively, while those obtained from the control group were: $0.541,0.330$, and 0.237 , respectively. Disability weights had a positive correlation with AUD severity levels. Employment status and family income were significantly associated with VAS disability weight among the control group.

Conclusion: The use of three different instruments to calculate disability weights for people with AUD is feasible in Thailand. The disability weights differ depending on the valuation methods used and respondent groups.

Keywords: alcohol-use disorders, disability weight, EQ-5D, time trade-off, visual analogue scale

Contact: Prof. Sawitri Assanangkornchai, M.D., Ph.D.

Department of Epidemiology, Faculty of Medicine, Prince of Songkla University, Hat Yai,

Songkhla 90110, Thailand.

E-mail: savitree.a@psu.ac.th

(c) 2020 JHSMR. Hosting by Prince of Songkla University. All rights reserved.

This is an open access article under the CC BY-NC-ND license

(http://www.jhsmr.org/index.php/jhsmr/about/editorialPolicies\#openAccessPolicy).
J Health Sci Med Res 2021;39(2):101-113 doi: $10.31584 /$ jhsmr.2020759 www.jhsmr.org 


\section{Introduction}

Alcohol consumption is a significant risk factor for the global burden of disease, and it causes substantial health loss. In 2016, the global prevalence of alcohol use was $2.2 \%$ in females and $6.8 \%$ in males, making it the seventh leading risk factor for disability adjusted life years (DALYs). Among people aged 15 to 49 years, $12.2 \%$ of male and $3.8 \%$ of female deaths were attributable to alcohol consumption in addition to $8.9 \%$ of male and $2.3 \%$ of female DALYs. ${ }^{1}$

Based on a study by the Thai Burden of Disease (BOD) Study Group, the total DALYs attributable to alcohol use disorders (AUD) in 2015 accounted for 12.0\% and $1.3 \%$ of the total burden of diseases among Thai males and females, respectively. Of all DALYs attributed to AUD, $13.1 \%$ and $1.7 \%$ were from years lived with disability (YLD) in males and females, respectively. ${ }^{2}$ A study investigating the burden of disease attributable to risk factors in 2014 showed that the leading risk factor was alcohol use, which accounted for 21,843 deaths $(12.0 \%){ }^{3}$

DALY is a composite indicator used to measure the magnitude of different health problems. It combines both aspects of morbidity and mortality in terms of years of life lost (YLL), and YLD. YLL reflects premature mortality due to a health condition, whereas YLD represents the time period living with a disability caused by the same condition. YLD is calculated based on the incidence of a health condition, its severity, age at onset, and duration of disability. A significant component of YLD is its "disability weight", which quantifies the severity of disease as a percentage of reduction from full health to death, with values ranging from 0 to 1 , wherein 0 represents full health, and 1 indicates death. ${ }^{4}$

There are various valuation methods used to calculate disability weight. In some instances, 'a health utility score' is measured first, either directly or indirectly, through the quantification of its perceived individual components. ${ }^{5}$ The utility score can then be converted into a disability weight based on the approach of each valuation method. Some valuation methods require respondents to make trade-offs, either in time (time trade-off; TTO) ${ }^{6}$ or person (person trade-off; PTO). ${ }^{7}$ Other methods, such as the European Quality of Life-5 Dimension (EQ-5D) scale and health utility index, use a multi-attribute system, which requires respondents to summarize their specific health conditions. ${ }^{8-10}$ Other methods use self-rated valuation on a visual analog scale (VAS), from best imaginable to the worst imaginable. ${ }^{11}$ Additionally, another method requires respondents to make a decision between pairs of health states, which is known as the paired-comparison method. $^{12}$ Thus, different methodological approaches, in terms of either source of population or procedure, can result in different estimates of disability weight, even with the same health status. Examples of this are the Global Burden of Disease (GBD) study ${ }^{13}$ and a Korean study $^{14}$, which used the paired comparison method among the general population and physicians but produced different disability weights for AUD (0.467 and 0.350).

The Thai BOD Study Group has used the disability weights from the GBD study to calculate DALYs for the Thai population since 1999. ${ }^{15}$ However, cultural differences between countries due to differences in social perspectives on disability and differences in the countries' health system may affect the relevance of the values used in such calculation. It might be possible that the disability weight of AUD in Thailand would be high due to high social stigma towards drinking and drinking problems. In Thailand, there have been several active campaigns to promote alcohol abstinence; an example is the "Stop Drinking during Buddhist Lent" campaign, which occurs every year. Alcohol-related problems, which may include domestic violence, aggressive behavior towards others and road 
traffic injuries that might affect communities at large, are considered as not only individual problems but also social ones. Community involvement in the prevention and management of alcohol-related problems is strongly encouraged, especially since an individual with alcohol dependence is regarded as a sick and somewhat morally-defective person. These could create high social judgement towards AUD, leading to higher disability scores among AUD patients perceived by both patients themselves and the general population.

As stated before, AUD is a leading cause of DALY in Thailand, yet no study has endeavored to explore the disability weight of AUD specifically. However, one study used $E Q-5 D$ to derive disability weights for five diseases: HIV/AIDs, diabetes mellitus, liver cancer, stroke, and trauma injuries. ${ }^{16}$ Thus, a strong need exists to the study disability weights of AUD and the methods to derive such values, which can be used in future studies.

This study aimed to determine the disability weights for AUD using different valuation methods: the EQ-5D scale, a VAS, and the TTO technique. We studied two groups of participants: (1) AUD patients, and (2) a control group consisting of non-AUD patients and their relatives/friends. Due to the lack of a gold standard for calculating the disability weights for $A \cup D^{14}$, our study's aim was not to identify the most valid method, but rather to compare different methods for calculating disability weights. Our study will help determine the feasibility of using these methods in Thai populations and provide preliminary results regarding disability weights of AUD for future studies in Thailand.

\section{Material and Methods}

A cross-sectional study was conducted from February to July 2018 in a public psychiatric hospital, a general hospital, and a community health center in Songkhla province, southern Thailand. Group 1 (AUD patients) included 150 out-patients aged 18 years or over and diagnosed with harmful use of alcohol (ICD10 code F10.1), alcohol dependence (F10.2), or unspecified mental and behavioral disorders due to the use of alcohol (F10.9), who visited the study hospitals during the study period. Those who had a tendency to have violent or aggressive behavior and/or severe cognitive impairment were excluded. Firstly, respondents were screened by a registered nurse from the outpatient clinics. If respondents were eligible, they were informed about the study protocol and then invited to participate in the study. Respondents were consecutively recruited until the required sample size was reached. Participating patients were classified into three severity levels based on their scores of the 10item Alcohol Use Disorder Identification Test ${ }^{17}$ (AUDIT; $<7=$ low risk (mild), 8-19=medium risk (moderate), and $>20=$ high risk (severe), respectively). The response rate was $79.5 \%$.

Group 2 contained 150 individuals, who were either patients with various non-communicable diseases such as diabetes mellitus and hypertension and patients exhibiting symptoms of influenza that attended the general hospitals or community health center during the study period, or their relatives and friends who were accompanying them. Those with a history of AUD, whose reason for the hospital visit was to seek help for their alcohol use problem, or who had been diagnosed with AUD by a medical doctor were excluded. The participants were invited by a research assistant, and all of them provided written informed consent before joining the study. The response rate for this group was $89.0 \%$.

\section{Valuation methods}

\section{Time trade-off measurement}

The TTO method is a health state valuation technique, which elicits scores in terms of preferences for a certain health state presented to the participant. The 
classical method allows participants to make a trade-off between length of life and quality of life. ${ }^{18}$ In this study, we assessed only the health state of better than death, which represented positive values. The disease duration was limited to 10 years, with the response scale being years lived in full health as the anchoring state. The TTO questions started with a comparison of 10 years living in each AUD severity state to $x(0<x<10)$ years in full health. Participants were asked to indicate at which point they were indifferent between the two health states_living with AUD for 10 years and living in full health for a shorter period of time. The questions were phrased so that death would follow at the end of each health state. ${ }^{18}$ An example of such a question is, "Choice $\boldsymbol{A}$ : stay in a mild AUD state for 10 years, then die versus Choice B: stay in full health for $x$ years $(0<x<10)$, then die." The time $(x)$ was initially set to zero years and then increased by one year until the respondents became indifferent between the two given choices. TTO visual aids were applied to visualize the trade-offs to help participants reach their point of indifference. The health state descriptions of mild, moderate, and severe AUD are presented in Table $1 .{ }^{4}$

\section{European Quality of Life and Visual Analog}

\section{Scale}

The Thai version of the EQ-5D instrument, a measure of health-related quality of life, was used in this study. ${ }^{19}$ The five dimensions were: mobility, self-care, active daily life, pain/discomfort, and anxiety/depression. We used the EQ-5D-5L version of the instrument, where each dimension is rated on five levels of difficulty: none, slight, moderate, severe, and extreme. ${ }^{19}$

Regarding the VAS, participants were asked to rate an AUD health state on a vertical line, where the endpoints were labeled, 'the best health you can imagine' and 'the worst health you can imagine', which were assigned as anchoring points. The VAS score was obtained by dividing the number marked on the scale by 100 , providing a score ranging from 0 to 1 .

All interviewers were Bachelor's degree graduates in health-related sciences. They were well-trained on how to use the valuation tools and the techniques of face-to-face interviews by the researcher and her supervisor. Moreover, a pilot study was conducted to evaluate the tools and questionnaires. A face-to-face

Table 1 Lay descriptions for severity state of alcohol use disorders in the study

\begin{tabular}{ll}
\hline AUD severity & Lay description \\
\hline Mild & Drinks a lot of alcohol and sometimes has difficulty controlling the urge to drink. While intoxicated, the person \\
& has difficulty performing daily activities. \\
Moderate & Drinks a lot, gets drunk almost every week, and has great difficulty controlling the urge to drink. Drinking and \\
& recovering cause great difficulty in daily activities, sleep loss, and fatigue. \\
Gevere & Drinking and recovering replace most daily activities. The person has difficulty thinking, remembering, and \\
& communicating, and feels constant pain and fatigue.
\end{tabular}

$\mathrm{AUD}=$ alcohol use disorders 
interview was conducted with the participants while they were waiting to see the doctor. Participants were initially asked about their personal characteristics (gender, age, education level, income, and number of family members). All participants were randomly assigned using random numbers to complete either the TTO or the EQ-5D-5L instruments, but all participants from both groups completed the VAS. For an AUD patient, after confirming his/her AUD level (mild, moderate, or severe), the interviewer first read to the patient the description of the AUD level corresponding to their current state of health and then asked him/her to complete the VAS and either the TTO or EQ-5D-5L instrument based on that health state. For the non-AUD group, the interviewer first read the description of an AUD level (mild, moderate, or severe) to the participant depending on whichever one was randomly assigned to him/her after recruitment, and then asked him/her to imagine being in that AUD state and complete the VAS and either the TTO or EQ-5D-5L instrument based on that given AUD condition. In summary, all 150 AUD patients rated their current AUD state on the VAS and then half rated their state on the TTO and the half on the EQ-5D-5L instrument. Similarly, 150 non-AUD patients rated a scenario-based AUD state that was randomly assigned to them on the VAS, and half of them rated the same state either on the TTO or the EQ-5D-5L, respectively. This resulted in about one-third of participants in each group rating a mild, moderate, or severe AUD level. Each participant completed each instrument on the AUD condition only once. Finally, the participants were asked if it was difficult to rate disability weight (a yes/no question) and provide reasons for the answer. The interviewing time for each participant was approximately 15 to 35 minutes.

The study was approved by the Ethical Review Committee for Research in Human Subjects, Faculty of
Medicine, Prince of Songkhla University (Rec: 60-35918-1).

\section{Statistical analysis}

\section{Disability weight estimation}

The utility score from the TTO instrument was calculated by dividing the duration of the state of full health at the point of indifference by the duration of the AUD state as follows:

$u(A)=\frac{x}{t}$

where $u(A)=$ utility of AUD

$x=$ time lived with full health

t=time lived with AUD state

The utility score was transformed to a disability weight by subtracting it from 1 .

The Thai version of the EQ-5D instrument was used. The participant was asked to rate his/her own or a scenario-based AUD state on each of the five dimensions on five levels of difficulty as stated above. Based on Pattanaphesaj's study among a Thai population, a coefficient specific to each level of difficulty was provided for all five dimensions. ${ }^{19}$ A single utility score ranging from 0 to 1 was calculated by subtracting the coefficients of the five dimensions of that person's health state from 1. The Thai EQ-5D index scores range from -0.4212 to 1.000, wherein 1 and 0 represent perfect health and death, respectively, and a score of less than 0 means a condition worse than death. Thereafter, the disability weight can be derived from this utility score, based on an algorithm of a simple linear regression as follows: ${ }^{16}$

Disability weight $=0.703631+(-0.703631 \times$ utility scores)

For the VAS, a participant's disability weight was calculated by subtracting their VAS score from 1. 


\section{Descriptive and analytical analysis}

The mean disability weights were presented for each valuation method and compared within and across participant groups using a paired or independent samples t-test. A linear regression was used to determine the associations between disability weights and respondent characteristics. Statistical significance was defined as a $\mathrm{p}$-value less than 0.05 .

\section{Results}

AUD patients tended to be younger, predominantly male, hold blue-collar jobs, and have higher levels of education and monthly family income compared to the non-AUD group. Chronic diseases were more common in the non-AUD group. There were no significant differences between the participants rating the TTO, VAS and EQ-5D instruments in terms of demographic characteristics (Table 2). Of all AUD patients, 72, 60, and 18 were diagnosed with F10.1, F10.2 and F10.9, with 37, 56, and 57 being in the mild, moderate and severe AUD levels, respectively. Of all non-AUD respondents, 49, 51, and 50 rated mild, moderate and severe AUD health states, respectively.

\section{Disability weights}

The TTO method took the longest time in responding to the questions, and one third of respondents $(30.7 \%)$ found it difficult to decide on the point of indifference between times in full health and with disease (Table 3 ).

A comparison of disability weights between three valuation methods within the same respondent group showed that the VAS gave the highest disability weights for both AUD (0.485) and non-AUD (0.541) groups, whereas the EQ-5D yielded the lowest weight values (0.311 and 0.237 in AUD and non-AUD groups, respectively;
Table 4). There were significant differences in the disability weights between different methods among AUD patients: TTO vs. EQ-5D (p-value=0.013), TTO vs. VAS ( $p-$ value= 0.036 ), and $E Q-5 D$ vs. VAS ( $p-v a l u e=0.001$ ). No significant differences in disability weights between TTO and EQ$5 D$ were seen among the non-AUD group ( $p$-value=0.107).

Comparing between respondent groups on the same method, based on the VAS, the mean disability weight obtained from non-AUD respondents was not significantly higher than that from the AUD patients, and the same can be said for the mean TTO and EQ-5D disability weights from the AUD patients compared to those from the non-AUD respondents.

The disability weight had a positive correlation with AUD severity levels (Table 5). In both groups of respondents, the disability weights, obtained from all methods, were higher for the more severe AUD conditions. Within the same severity level, the VAS provided the highest values of all methods. No significant differences in the disability weights between all three methods were found in severe AUD levels, for both AUD and non-AUD patients (all p-values>0.05).

In regard to the VAS instrument, the multiple linear regression models showed that, among the AUD group, an increase of one family member was significantly related to a decrease of disability weight by an average of 0.025 points. Among the non-AUD group, the disability weight for an unemployed person was, on average, significantly higher (by 0.092 points) than for those who were employed. Similarly, an increase of family income by one thousand Thai baht resulted in a significant increase in average disability weights (by 0.006 points) while holding all other variables constant (Table 6). 


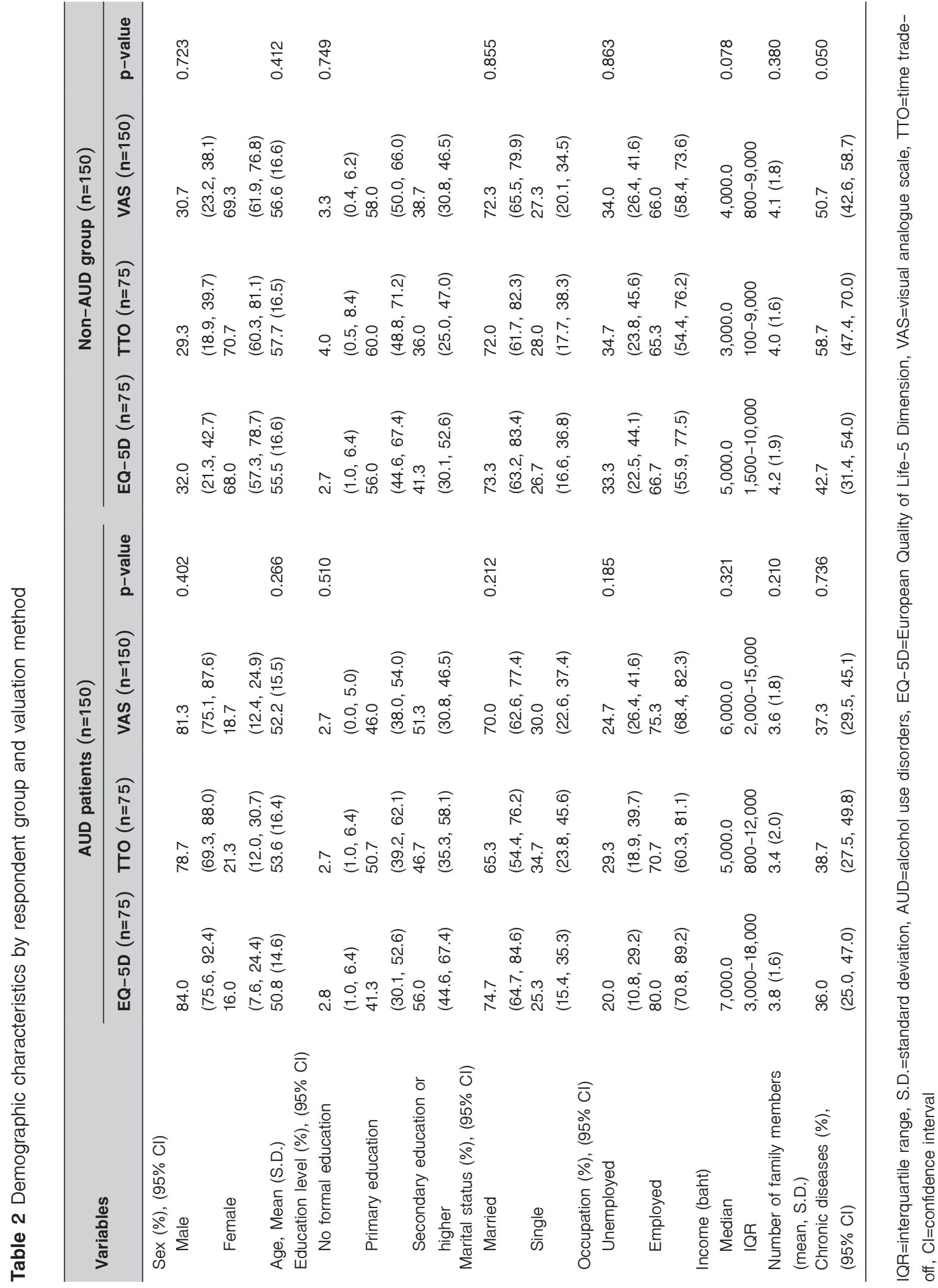


Table 3 Difficulty in rating, response time, and disability weights for alcohol use disorders based on three valuation methods, stratified by respondent group

\begin{tabular}{|c|c|c|c|}
\hline Attribute & VAS & TTO & EQ-5D \\
\hline Difficulty in rating $^{\dagger}(\%),(95 \% \mathrm{Cl})$ & $\begin{array}{l}4.3 \\
(2.0-6.7)\end{array}$ & $\begin{array}{l}30.7 \\
(23.2-38.1)\end{array}$ & $\begin{array}{l}5.3 \\
(1.7-9.0)\end{array}$ \\
\hline Response time (min), mean (S.D.) & $1.5(0.3)$ & $8.9(4.3)$ & $5.6(-3.6)$ \\
\hline
\end{tabular}

${ }^{\dagger}$ Percentage of respondents who found it difficult to rate the scale or decide on the point of indifference.

VAS=visual analogue scale, TTO=time trade-off, EQ-5D=European Quality of Life-5 Dimension, $\mathrm{Cl}=$ confidence interval, $A U D=a l c o h o l ~ u s e$ disorders, S.D.=standard deviation

Table 4 Disability weights for alcohol use disorders for three valuation methods stratified by respondent group

\begin{tabular}{lllllll}
\hline \multirow{2}{*}{ Valuation method } & \multicolumn{2}{c}{ AUD patients } & & \multicolumn{2}{c}{ Non-AUD group } \\
\cline { 2 - 3 } \cline { 5 - 6 } & Mean (S.D.) & Range & & Mean (S.D.) & Range \\
\hline VAS & $0.485(0.231)$ & $0.000-1.000$ & & $0.541(0.230)$ & $0.100-1.000$ \\
TTO & $0.405(0.330)$ & $0.000-1.000$ & & $0.330(0.316)$ & $0.000-1.000$ \\
EQ-5D & $0.311(0.271)$ & $0.000-0.727$ & & $0.237(0.227)$ & $0.000-0.883$ \\
\hline
\end{tabular}

AUD=alcohol use disorders, S.D.=standard deviation, VAS=visual analogue scale, TTO=time trade-off, EQ-5D=European Quality of Life-5 Dimension

Table 5 Comparison of disability weights for alcohol use disorders based on three valuation methods stratified by severity level

\begin{tabular}{|c|c|c|c|c|c|c|}
\hline \multirow{2}{*}{ Valuation method } & \multicolumn{3}{|c|}{ AUD patients } & \multicolumn{3}{|c|}{ Non-AUD group } \\
\hline & Mild & Moderate & Severe & Mild & Moderate & Severe \\
\hline VAS & $0.395(0.213)$ & $0.511(0.153)$ & $0.546(0.282)$ & $0.441(0.192)$ & $0.555(0.150)$ & $0.624(0.291)$ \\
\hline TTO & $0.212(0.256)$ & $0.252(0.241)$ & $0.450(0.262)$ & $0.111(0.132)$ & $0.232(0.196)$ & $0.369(0.261)$ \\
\hline$E Q-5 D$ & $0.318(0.386)$ & $0.398(0.319)$ & $0.498(0.261)$ & $0.215(0.154)$ & $0.377(0.331)$ & $0.392(0.389)$ \\
\hline
\end{tabular}

Numbers in table show means with standard deviations in brackets.

$\mathrm{AUD}=$ alcohol use disorders, VAS=visual analogue scale, TTO=time trade-off, EQ-5D=European Quality of Life-5 Dimension 
Table 6 Multivariate linear regression model predicting alcohol use disorder disability weights based on the visual analogue scale method

\begin{tabular}{|c|c|c|c|c|c|c|}
\hline \multirow{3}{*}{$\begin{array}{l}\text { Variable } \\
\text { Sex }(\text { female })^{\dagger}\end{array}$} & \multicolumn{3}{|c|}{ AUD patients } & \multicolumn{3}{|c|}{ Non-AUD group } \\
\hline & \multirow{2}{*}{$\begin{array}{l}\text { Estimate } \\
0.070\end{array}$} & \multicolumn{2}{|c|}{$95 \% \mathrm{Cl}$} & \multirow{2}{*}{$\begin{array}{l}\text { Estimate } \\
-0.051\end{array}$} & \multicolumn{2}{|c|}{$95 \% \mathrm{Cl}$} \\
\hline & & -0.033 & 0.173 & & -0.135 & 0.033 \\
\hline Age & -0.002 & -0.005 & 0.001 & 0.002 & -0.001 & 0.005 \\
\hline Education (secondary school) $^{\dagger}$ & 0.011 & -0.088 & 0.110 & 0.091 & -0.014 & 0.196 \\
\hline Marital status (married $)^{\dagger}$ & 0.087 & -0.002 & 0.176 & 0.034 & -0.052 & 0.121 \\
\hline Employment status (unemployed) ${ }^{\dagger}$ & 0.011 & -0.085 & 0.108 & $0.092^{*}$ & 0.005 & 0.179 \\
\hline Income (per 1,000 baht) & -0.001 & -0.005 & 0.002 & $0.006^{*}$ & 0.000 & 0.012 \\
\hline Family size & $-0.025^{\star}$ & -0.047 & -0.003 & -0.012 & -0.034 & 0.010 \\
\hline Chronic disease(s) (yes/no) & -0.003 & -0.086 & 0.079 & 0.052 & -0.031 & 0.135 \\
\hline Past-year hospitalization (yes/no) & -0.052 & -0.134 & 0.030 & -0.051 & -0.125 & 0.022 \\
\hline Past-year accident (yes/no) & -0.001 & -0.082 & 0.080 & 0.004 & -0.080 & 0.088 \\
\hline Intercept & 0.535 & 0.304 & 0.767 & 0.432 & 0.153 & 0.712 \\
\hline
\end{tabular}

${ }^{\dagger}$ Reference groups for categorical variables are as follows: sex (male), education (less than primary school), marital status (single), employment status (employed).

$\mathrm{Cl}=$ confidence interval, $\mathrm{AUD}=$ alcohol use disorders, ${ }^{*} \mathrm{p}$-value $<0.05$

\section{Discussion}

This study compare the disability weights of alcohol use disorders calculated using three different valuation methods: EQ-5D, TTO, and VAS. The disability weights calculated using the VAS method among the non-AUD respondents $(0.44,0.56$ and 0.62 for mild, moderate, and severe AUD, respectively) were comparable with those from a Korean study, which used a pairwise method among the general population $(0.47,0.49$ and 0.56$) .{ }^{20}$ Furthermore, the TTO method in our study elicited disability weights for mild (0.22) and moderate (0.38) AUD, which were similar to those obtained from the pairwise method in the GBD study $^{4}$ (0.24 and 0.37); however, the severe AUD disability weight was lower in our study (0.39 vs. 0.57). As different valuation methods were used, it is difficult to explain if the differences were solely because of methodological discrepancy and/or due to the effects of other social and environmental factors. Dolan and Sutton argued that the quality of life results vary more across cultures than across methodologies. ${ }^{11}$ In some cultures, the judgment of a good quality of life is based on what is good for the community, while in other cultures, a good life follows prescriptions in religion and tradition. ${ }^{21}$

Furthermore, as seen in our study, there was a significant relationship between disability weight based on the VAS and the demographic characteristics of respondents, indicating that those characteristics affected the ways the respondents gave values on their disability, which is in keeping with previous studies. ${ }^{62-24}$ In Thailand, families are largely closely-knit and extended, and the family plays a very important part in daily living and may be the sole source of social support to an AUD patient. Intergenerational relations between older-age parents and grandparents with their children and grandchildren may be the only asset the AUD patient possesses. This may explain the negative relationship between family size and disability weights derived from the AUD patients. Among the non-AUD participants, the reasons for their employment 
status and income being positively associated with disability weight are unclear. It could be that unemployed respondents might have compared the VAS score of AUD state with their own quality of life and tended to rate higher disability weights than did the employed respondents. However, since the estimate income level was low (0.006) and marginally significant $(95 \% \mathrm{Cl}$ : $0.000,0.012)$, it may not be that meaningful. Nevertheless, this reflects a need for more empirical evidence on the universality of disability weight calculations.

Evaluating the validity of disability weights is difficult, mainly due to the absence of a gold standard. ${ }^{25,26}$ Within our study, significant differences were found between scores derived by the VAS, TTO, and EQ-5D methods. This indicates the importance of methods in deriving disability weights. ${ }^{25}$ However, it is not clear why the VAS method gave the highest weights in this study. In fact, among the AUD patient group, it is possible that the patients' current health condition on the day of interview, rather than their overall AUD condition, affected the VAS score they provided. Likewise, when surveying the non-AUD group participants, it would be difficult for them to imagine the health condition of an AUD individual, and other factors such as their past experience, either positive or negative, and relationships with people with AUD could affect their attitudes towards AUD patients. ${ }^{27-29}$ These, in turn, may have been translated into the VAS scores they gave on the day of interview..$^{30,31}$

It has been found that the TTO method results in higher disability weights than the EQ-5D one ${ }^{32}$, which may be due to differences in methodological techniques as well as the concepts of the methods. ${ }^{33,34}$

The TTO theory describes the method of decisionmaking under uncertainty based on a set of rational behaviors ${ }^{5}$, whereas EQ-5D measures a person's preferences with respect to health, or how health is valued $^{19}$, using a summarized value of their complex health condition. For AUD, it is likely that living with this disease condition is preferable than death; therefore, the duration of time living in an AUD state, $t$, will be much greater than the duration of time living in full health, $x$. The utility value $(x / t)$ will, therefore, be smaller than one, resulting in a large disability weight $(1-x / t)$. In this respect, when thinking of an AUD condition, in terms of each domain of the $E Q-5 D$, it is most likely that both AUD and non-AUD patients would rate either no or very little problem for most and, perhaps, all domains. It could be that the EQ-5D is not sensitive enough for AUD patients in general, as most people with AUD do not have problems with their mobility, self-care, active daily life, pain/discomfort or anxiety/depression. The utility value obtained from the EQ-5D was thus high, resulting in a lower disability weight than the one calculated via the TTO method.

When comparing between AUD patients and participant in the non-AUD group, based on the VAS method, AUD patients provided lower mean disability weights for all severity levels. This might be explained in terms of their attitudes towards their own disease along with the treatments that they received. Due to their direct experience of illness, AUD patients may initially consider a particular disease condition as unpleasant, but then they become adapted to it and learn to tolerate it. ${ }^{35}$

On the other hand, AUD patients provided higher disability weights for the EQ-5D and TTO methods than those of the non-AUD group. Discrepancies in disability weights between patients and the general population, particularly in certain dimensions of health, have been shown. ${ }^{36-38}$ Some studies have suggested that patients consider anxiety/depression more problematic than other health problems. ${ }^{39,40}$ It may be that AUD patients imagine "non-tangible" dimensions of health states (anxiety or depression, and pain or discomfort) more accurately than the non-AUD group, who has less experience with those health states. ${ }^{38}$ Consequently, the overall disability 
weight among AUD patients, derived from EQ-5D, was higher than that of the control group.

In terms of feasibility, this study shows the amount of time respondents spent to complete the TTO tasks as being higher than those required to complete both the EQ5D and VAS tasks (9 $\mathrm{min} / T T O, 5.6 \mathrm{~min} / E Q 5 D$, and 1.5 min NAS tasks). Additionally, $31.0 \%$ of the TTO respondents found it difficult to decide upon where the point of indifference was for them. It is certain that the TTO requires the highest cognitive load compared to the other measurements. However, given the fact that it is the only method that indicates the value of the disability weight, based on the fundamentals of decision-making, under uncertainty situations, and it was feasible for use in our samples. This valuation method is, therefore, worth considering for use in future studies in Thailand.

There are many limitations to this study. Firstly, it was hospital-based, and conducted in only one province, limiting its generalizability to the whole country. Interviewer bias, socially desirable bias, and acquiescence bias may have occurred. For example, respondents often found it easier to agree than to disagree with their interviewer.

\section{Conclusion}

Our study indicates that disability weights of AUD differ significantly by valuation method and severity level of the illness but not by respondent group. We demonstrated that all three valuation methods were applicable among both Thai AUD patients and general lay people and provided good face validity. Socio-demographic and economic characteristics did not have much effect on the AUD patients' perception of their disability weight. Our estimated disability weights based on the VAS method among non-AUD participants are comparable with those reported by a Korean study, although based on different methods. It could, therefore, be concluded that disability weight does not depend on who values it but on the method from which it is derived; thus, a national set of disability weights should be studied in favor of burden of disease study in Thailand. Future studies should consider the systematic assessment of the validity of tools to be used in the Thai population and also involve larger groups of subjects in order to generalize for a general population group and keep the random measurement error at an acceptable level. Moreover, different types of illnesses and disease-specific factors should be investigated further in terms of the variations of their health states.

\section{Acknowledgement}

We wish to thank the Centre for Alcohol Studies for the research grant, and the patients and staff at the hospitals involved for their participation in the study as well as Mr. Edward McNeil for his assistance with correcting the English maunscript.

\section{Funding sources}

This study was supported by the Centre for Alcohol Studies grant \#60-C6-0001.

\section{Conflict of interest}

The authors declare no conflict of interest.

\section{References}

1. Griswold MG, Fullman N, Hawley C, Arian N, Zimsen SRM, Tymeson HD, et al. Alcohol use and burden for 195 countries and territories, 1990-2016: a systematic analysis for the Global Burden of Disease Study 2016. Lancet 2018;392: 1015-35.

2. Burden of diseases Thailand study group. Burden of diseases and Injuries of Thailand 2015. Nonthaburi: International Health Policy Program; 2017.

3. Burden of diseases Thailand study group. Thailand burden of diseases attributable to risk factors 2014. Nonthaburi: International Health Policy Program; 2018. 
4. Salomon JA, Haagsma JA, Davis A, de Noordhout CM, Polinder S, Havelaar AH, et al. Disability weights for the Global Burden of Disease 2013 study. Lancet Glob Health 2017;3: e712-23.

5. Torrance GW. Utility approach to measuring health-related quality of life. J Chronic Dis 1987;40:593-603.

6. Dolan P, Gudex C, Kind P, Williams A. The time trade-off method: results from a general population study. Health Econ 1996;5:141-54.

7. Nord E. The person-trade-off approach to valuing health care programs. Med Decis Making 1995;15:201-8.

8. Maheswaran H, Petrou S, Rees K, Stranges S. Estimating $E Q-5 D$ utility values for major health behavioural risk factors in England. J Epidemiol Community Health 2013;67:172-80.

9. Chavez LJ, Bradley K, Tefft N, Liu CF, Hebert P, Devine B. Preference weights for the spectrum of alcohol use in the U.S. Population. Drug Alcohol Depend 2016;161:206-13.

10. Günther $\mathrm{O}$, Roick $\mathrm{C}$, Angermeyer MC, König HH. The EQ-5D in alcohol dependent patients: relationships among healthrelated quality of life, psychopathology and social functioning. Drug Alcohol Depend 2007;86:253-64.

11. Dolan P, Sutton M. Mapping visual analogue scale health state valuations onto standard gamble and time trade-off values. Soc Sci Med 1997;44:1519-30.

12. Rehm J, Frick U. Establishing disability weights from pairwise comparisons for a US burden of disease study. Int J Methods Psychiatr Res 2013;22:144-54.

13. Haagsma JA, Maertens de Noordhout C, Polinder S, Vos $\mathrm{T}$, Havelaar AH, Cassini A, et al. Assessing disability weights based on the responses of 30,660 people from four European countries. Popul Health Metr 2015;13:10.

14. Ock M, Lee JY, Oh IH, Park H, Yoon SJ, Jo MW. Disability Weights Measurement for 228 Causes of Disease in the Korean Burden of Disease Study 2012. J Korean Med Sci 2016; 31(Suppl 2):S129-38.

15. Bundhamcharoen K, Odton P, Phulkerd S, Tangcharoensathien V. Burden of disease in Thailand: changes in health gap between 1999 and 2004. BMC Public Health 2011;11:53.

16. Burden of diseases Thailand study group. Thai disability weight 2009. Nonthaburi: International Health Policy Program; 2016.

17. Babor TF, Higgins-Biddle JC, Saunders JB, Monteiro MG.
The alcohol use disorders identification test: guidelines for use in primary care. $2^{\text {nd }}$ ed. Geneva: WHO; 2014.

18. Craig BM, Oppe M. From a different angle: a novel approach to health valuation. Soc Sci Med 2010;70:169-74.

19. Pattanaphesaj J, Thavorncharoensap M, Ramos-Goñi JM, Tongsiri S, Ingsrisawang L, Teerawattananon Y. The EQ-5D-5L Valuation study in Thailand. Expert Rev Pharmacoecon Outcomes Res 2018;18:551-8.

20. Ock M, Ahn J, Yoon SJ, Jo MW. Estimation of disability weights in the general population of South Korea using a paired comparison. PLOS ONE 2016;11. doi: 10.1371/journal. pone.0162478

21. Arnesen TM, Norheim OF. Quantifying quality of life for economic analysis: time out for time tradeoff. Med Humanit 2003;29: 81-6.

22. Arnesen T, Nord E. The value of DALY life: problems with ethics and validity of disability adjusted life years. BMJ 1999;319: 1423-5.

23. Lundberg L, Johannesson M, Isacson DGL, Borgquist L. Healthstate utilities in a general population in relation to age, gender and socioeconomic factors. Eur J Public Health 1999;9:211-7.

24. Dolan P, Roberts J. To what extent can we explain time trade-off values from other information about respondents? Soc Sci Med 2002;54:919-29.

25. Haagsma JA, Polinder S, Cassini A, Colzani E, Havelaar AH. Review of disability weight studies: comparison of methodological choices and values. Popul Health Metr 2014;12:20.

26. Ock M, Park B, Park H, Oh IH, Yoon SJ, Cho B, et al. Disability weights measurement for 289 causes of disease considering disease severity in Korea. J Korean Med Sci 2019;34(Suppl 1). doi: 10.3346/jkms.2019.34.e60.

27. Ock M, Ko S, Lee HJ, Jo MW. Review of issues for disability weight studies. Health Policy Manag 2016;26:352.

28. Crisp AH, Gelder MG, Rix S, Meltzer HI, Rowlands OJ. Stigmatisation of people with mental illnesses. $\mathrm{Br} \mathrm{J}$ Psychiatry 2000;177:4-7.

29. Keyes KM, Hatzenbuehler ML, McLaughlin KA, Link B, Olfson M, Grant BF, et al. Stigma and treatment for alcohol disorders in the United States. Am J Epidemiol 2010;172:1364-72.

30. Badia X, Monserrat S, Roset M, Herdman M. Feasibility, validity and test-retest reliability of scaling methods for health states: the visual analogue scale and the time tradeoff. Qual Life Res 1999;8:303-10. 
31. Jelsma J, Ferguson G. The determinants of self-reported health-related quality of life in a culturally and socially diverse South African community. Bull World Health Organ 2004;82: 206-12.

32. Torrance GW, Feeny D. Utilities and quality-adjusted life years Int J Technol Assess Health Care 1989;5:559-75.

33. Tijhuis G, Jansen S, Stiggelbout A, Zwinderman A, Hazes J, Vlieland $T$. Value of the time trade off method for measuring utilities in patients with rheumatoid arthritis. Ann Rheum Dis 2000;59:892-7.

34. Attema AE, Edelaar-Peeters $Y$, Versteegh MM, Stolk EA. Time trade-off: one methodology, different methods. Eur J Health Econ 2013;14(Suppl 1):S53-64.

35. Boyd NF, Sutherland HJ, Heasman KZ, Tritchler DL, Cummings BJ. Whose utilities for decision analysis? Med Decis Making 1990;10:58-67.

36. Weyler EJ, Gandjour A. Empirical validation of patient versus population preferences in calculating QALYs. Health Serv Res 2011;46:1562-74.

37. Little MHR, Reitmeir P, Peters A, Leidl R. The impact of differences between patient and general population EQ5D-3L values on the mean tariff scores of different patient groups. Value Health 2014;17:364-71.

38. Ogorevc M, Murovec N, Fernandez NB, Rupel VP. Questioning the differences between general public vs. patient based preferences towards EQ-5D-5L defined hypothetical health states. Health Policy 2019;123:166-72.

39. Mann R, Brazier J, Tsuchiya A. A comparison of patient and general population weightings of EQ-5D dimensions. Health Econ 2009;10:363-72.

40. Burstrom K, Sun S, Gerdtham UG, Henriksson M, Johannesson M. Swedish experience-based value sets for EQ-5D health states. Qual Life Res 2014;12:431-42. 